Iberian Conference on Information Systems and Technologies, CISTIVolume 2018June, 27 June 2018, Pages 1-613th Iberian Conference on Information Systems and Technologies, CISTI 2018; Caceres; Spain; 13 June 2018 through 16 June 2018; Category numberCFP1803K-ART; Code 137540

\title{
Wearable technology model to control and monitor hypertension during pregnancy(Conference Paper)
}

- $\quad$ Lopez, B.D.B. ${ }^{\mathrm{a} E m a i l}$ Author,

- $\quad$ Aguirre, J.A.A. ${ }^{\mathrm{a}}$ Email Author,

- Coronado, D.A.R. ${ }^{a}$ Email Author,

- Gonzalez, P.A. ${ }^{\text {bEmail Author }}$

- $\quad{ }^{a}$ Escuela de Ingeniería de Sistemas y Computación, Universidad Peruana de Ciencias Aplicadas, Lima, Peru

- ${ }^{b}$ Rowe School of Business, Faculty of Management, Dalhousie University, Halifax, Canada

\begin{abstract}
View references (27)
In this paper, we proposed a wearable technology model to control and monitor hypertension during pregnancy. We enhanced prior models by adding a series of health parameters that could potentially prevent and correct hypertension disorders in pregnancy. Our proposed model also emphasizes the application of real-time data analysis for the healthcare organization. In this process, we also assessed the current technologies and systems applications offered in the market. The model consists of four phases: 1 . The health parameters of the patient are collected through a wearable device; 2 . The data is received by a mobile application; 3 . The data is stored in a cloud database; 4 . The data is analyzed on real-time using a data analytics application. The model was validated and piloted in a public hospital in Lima, Peru. The preliminary results showed an increased-on number of controlled patients by $11 \%$ and a reduction of maternal deaths by $7 \%$, among other relevant health factors that allowed healthcare providers to take corrective and preventive actions. (C) 2018 AISTI.
\end{abstract}

\section{Author keywords}

Data analyticsHypertensionMobile health applicationPregnancyTechnologyWearable

\section{Indexed keywords}

Engineering controlled Health careHospitalsInformation systemsInformation terms: useObstetrics

Engineering uncontrolled Data analyticsHypertensionMobile health terms applicationPregnancyWearable

Engineering main heading:

Wearable technology 
- ISSN: 21660727

- ISBN: 978-989984348-6

- Source Type: Conference Proceeding

- Original language: English

- DOI: $10.23919 /$ CISTI.2018.8399200

- Document Type: Conference Paper

- Volume Editors: Rocha A.,Cota M.P.,Lozano-Tello A.,Goncalves R.

- Sponsors: IEEE Portugal Section,IEEE SMC Portugal Chapter,IEEE Spain Section

- Publisher: IEEE Computer Society 